\title{
Tempo entre o início dos sintomas e o tratamento de tuberculose pulmonar em um município com elevada incidência da doença*
}

\author{
Time from symptom onset to the initiation of treatment of pulmonary \\ tuberculosis in a city with a high incidence of the disease \\ Marina de Loureiro Maior, Renata Leborato Guerra, \\ Michelle Cailleaux-Cezar, Jonathan Eric Golub, Marcus Barreto Conde
}

\begin{abstract}
Resumo
Objetivo: Estimar o tempo entre o início dos sintomas e o início do tratamento de pacientes com tuberculose pulmonar virgens de tratamento e com resultado positivo na baciloscopia direta do escarro, assim como avaliar as variáveis associadas à demora no diagnóstico e no início do tratamento. Métodos: Estudo descritivo exploratório em pacientes virgens de tratamento para tuberculose, com idade $\geq 12$ anos e resultado positivo para BAAR no escarro. Entre 2006 e 2008, os 199 pacientes incluídos no estudo foram entrevistados, e seus exames complementares foram revisados no momento da consulta para o início de tratamento para tuberculose em uma unidade básica de saúde no município de Nova lguaçu (RJ). Resultados: As medianas (e seus respectivos intervalos interquartílicos) para o tempo entre o início dos sintomas e o início do tratamento, o tempo até a procura por atendimento médico, o tempo até o diagnóstico e o tempo até o início do tratamento, em semanas, foram, respectivamente, 11 (6-24), 8 (4-20), 2 (1-8) e 1 (1-1).As variáveis gênero, idade, escolaridade, uso prévio de antibióticos, status HIV, local da primeira consulta médica e extensão radiológica da doença não se associaram ao tempo até o diagnóstico ou ao tempo até o início do tratamento. A principal razão para a demora dos pacientes em procurar o serviço de saúde foi sua dificuldade em reconhecer seus sintomas como indicativos de doença. Conclusões: Os tempos até o diagnóstico e até o início do tratamento para tuberculose foram inaceitavelmente longos na amostra estudada.
\end{abstract}

Descritores: Tuberculose/diagnóstico; Tuberculose/terapia; Diagnóstico tardio.

\begin{abstract}
Objective: To estimate the time elapsed between the onset of symptoms and the initiation of treatment of pulmonary tuberculosis among treatment-naïve patients with positive results in sputum smear microscopy, and to evaluate the variables associated with delays in diagnosis and in treatment initiation. Methods: This was a descriptive exploratory study involving 199 treatment-naïve tuberculosis patients $\geq 12$ years of age with AFB-positive sputum smear microscopy results between 2006 and 2008. At their first (treatment initiation) visit to a primary health care clinic in the city of Nova lguaçu, Brazil, the patients were interviewed and their ancillary test results were reviewed. Results: The medians (and respective interquartile ranges) of the time from symptom onset to the initiation of treatment of pulmonary tuberculosis, from symptom onset to seeking medical attention, from entry into care to diagnosis, and from entry into care to treatment initiation, in weeks, were 11 (6-24), 8 (4-20), 2 (1-8), and 1 (1-1), respectively. The variables gender, age, level of education, previous use of antibiotics, HIV status, site of first medical visit, and radiological extent of tuberculosis showed no associations with the time from entry into care to diagnosis and to treatment initiation. The main reason for the delay in seeking medical attention reported by the patients was their inability to recognize their symptoms as indicators of a disease. Conclusions: Among the patients studied, there was an unacceptably long delay between the onset of symptoms and the initiation of tuberculosis treatment.
\end{abstract}

Keywords: Tuberculosis/diagnosis; Tuberculosis/therapy; Delayed diagnosis.

\footnotetext{
* Trabalho realizado no Instituto de Doenças do Tórax, Universidade Federal do Rio de Janeiro, Rio de Janeiro (RJ) Brasil. Endereço para correspondência: Marcus B. Conde. Laboratório de Pesquisa Clínica em Tuberculose. Instituto de Doenças do Tórax da UFRJ, Rua Professor Rodolpho Rocco, 255, prédio do HUCFF, 6º andar, Cidade Universitária, CEP 21941-913, Rio de Janeiro, RJ, Brasil.

Tel. 5521 2562-2432. Fax: 5521 2562-2853. E-mail: marcusconde@hucff.ufrj.br

Apoio financeiro: Esse estudo foi parcialmente financiado pela Fundação Carlos Chagas Filho de Amparo à Pesquisa do Estado do Rio de Janeiro (FAPERJ; E-26/101.491/2010 e E-26/171.505/2006). Marcus B. Conde é bolsista de produtividade científica do Conselho Nacional de Desenvolvimento Científico e Tecnológico (CNPq; n 300414/2010-2).

Recebido para publicação em 5/9/2011. Aprovado, após revisão, em 20/1/2012.
} 


\section{Introdução}

Apesar da redução progressiva nas taxas de incidência da tuberculose nos últimos 10 anos, o Brasil continua sendo um dos 22 países responsáveis por $80 \%$ dos casos de tuberculose em todo o mundo.(1) Em 2009, a Organização Mundial da Saúde (OMS) estimou uma taxa de incidência de 45 casos de tuberculose por 100.000 habitantes no Brasil e, de acordo com dados do Ministério da Saúde do Brasil, essa taxa foi de 37 casos por 100.000 habitantes no mesmo ano. $(1,2) 0$ Rio de Janeiro (71/100.000 habitantes) é o estado com a maior incidência de tuberculose do Brasil. Nova Iguaçu é o município com a terceira maior taxa de incidência (76/100.000 habitantes) do estado, logo após os municípios do Rio de Janeiro e de Caxias. Nova Iguaçu tem uma população estimada de 831.000 habitantes, um produto interno bruto de três bilhões de reais e um índice de desenvolvimento humano de 0,762, ficando na $17^{\circ}$ colocação entre os municípios com população total entre 500 mil e 1 milhão de habitantes do Brasil. Por ser a tuberculose uma doença transmissivel por via aérea, a quebra da cadeia de transmissão, através do diagnóstico precoce e do início imediato do tratamento, é um aspecto crucial na estratégia de controle da transmissão de Mycobacterium tuberculosis e do surgimento de novos casos da doença..$^{(1,3)}$

A principal estratégia para o diagnóstico de casos novos de tuberculose pulmonar recomendada pelo manual de tuberculose do Programa Nacional de Controle da Tuberculose no Brasil é a realização da baciloscopia direta do escarro para a pesquisa de BAAR em paciente "sintomático respiratório", definido como o indivíduo com tosse por três semanas ou mais.(2) Estudos realizados em diferentes cenários epidemiológicos já referiram que certos fatores, como a desinformação dos pacientes, a deficiência no conhecimento sobre a tuberculose dos profissionais da área de saúde, a falta de investimento em uma política de saúde pública organizada e a dificuldade dos pacientes no acesso aos serviços de saúde e na realização de exames, podem se associar ao retardo no diagnóstico da tuberculose. ${ }^{(4-7)}$

0 objetivo do presente estudo foi estimar o tempo entre o início dos sintomas e o início do tratamento de pacientes com tuberculose pulmonar virgens de tratamento e com resultado positivo de baciloscopia direta do escarro, assim como avaliar as variáveis associadas tanto à demora no diagnóstico quanto à demora no início do tratamento em um município com elevada taxa de incidência de tuberculose no estado do Rio de Janeiro.

\section{Métodos}

Estudo descritivo exploratório realizado em uma Unidade Pública de Saúde (UPS) de referência para tuberculose no município de Nova lguaçu, RJ, entre $1^{\circ}$ de junho de 2006 e 31 de julho de 2008. Foram convidados a participar do estudo todos os pacientes com idade $\geq 12$ anos, com resultado positivo na baciloscopia direta do escarro, virgens de tratamento para tuberculose, e que compareceram à consulta da UPS com o objetivo de iniciar o tratamento para tuberculose pulmonar. Todos os indivíduos (ou seus responsáveis legais, no caso de menores de 18 anos de idade) que assinaram o termo de consentimento livre e esclarecido foram submetidos a uma entrevista padronizada, utilizando um instrumento de coleta de dados pré-testado e elaborado especificamente para o presente estudo, o qual incluía perguntas sobre dados pessoais (idade, escolaridade, etc.) e variáveis relacionadas ao diagnóstico de tuberculose, como o tempo do início dos sintomas, o uso prévio de antibióticos, o status HIV, o local e a data da primeira consulta médica e a extensão radiológica da doença. Foi também perguntado aos sujeitos da pesquisa o motivo que os levou a procurar, ou não, o serviço de saúde. Os sujeitos de pesquisa tiveram ainda os resultados dos exames de escarro e as radiografias de tórax revisadas pela equipe do estudo. As radiografias de tórax foram analisadas de forma independente por dois pneumologistas mascarados quanto aos dados dos pacientes. Em caso de discordância do laudo final, um terceiro pneumologista avaliou a radiografia e definiu o laudo. Definiu-se como doença radiologicamente extensa, com base nas imagens radiográficas, a soma da área de acometimento igual ou maior do que um lobo inteiro ou a presença de uma ou mais cavitações cuja área total somasse pelo menos $2 \mathrm{~cm}$. Para todos os participantes, foi disponibilizada a realização do teste rápido para a detecção de HIV (Determine ${ }^{\circledR}$ HIV1/2 test; Abbott Brasil, Rio de Janeiro, Brasil), exceto para aqueles com um resultado de teste anti-HIV positivo previamente documentado. Os seguintes critérios de exclusão foram utilizados: tratamento prévio de tuberculose por pelo menos 7 dias, entrevista não 
concluída e ausência de resultado da baciloscopia do escarro. 0 estudo foi apresentado à direção da UPS e à coordenação do Programa de Controle de Tuberculose do município de Nova lguaçu e foi aprovado pelo Comitê de Ética em Pesquisa do Hospital Universitário Clementino Fraga Filho da Universidade Federal do Rio de Janeiro em 8 de maio de 2006 ( $n^{\circ}$ 447/06).

A análise estatística foi realizada utilizando o programa Statistical Package for the Social Sciences, versão 11.0 (SPSS Inc., Chicago, IL, EUA). As variáveis relacionadas a "tempo" foram medidas em semanas e descritas para as diferentes situações: a variável "tempo até a procura por atendimento" foi definida como o tempo entre a data referida pelo paciente como aquela do início dos sintomas e a data da primeira consulta em um serviço de saúde, "tempo até o diagnóstico" foi definida como o tempo entre a data da primeira consulta em um serviço de saúde e a data do resultado positivo da baciloscopia direta do escarro, e "tempo até o início do tratamento" foi definida como o tempo entre a data do resultado positivo da baciloscopia direta do escarro e a data da consulta para o início do tratamento. Outras variáveis analisadas foram 0 gênero, a idade, o grau de escolaridade, o uso prévio de antibióticos, o status HIV, o local da primeira consulta médica (unidade primária de saúde ou serviço de emergência) e a extensão radiológica da doença. A mediana e o intervalo interquartílico 25-75\% (11) das variáveis foram calculados, e os testes de Kruskal-Wallis e de Mann-Whitney foram utilizados para comparar as medianas entre os grupos de pacientes. A análise de regressão linear múltipla foi utilizada para identificar associações entre as variáveis independentes e as variáveis dependentes, sendo ajustada para possíveis variáveis de confusão. Os resultados foram considerados significativos quando $\mathrm{p}<0,05$.

\section{Resultados}

Foram incluídos no estudo 200 pacientes com resultado positivo para BAAR na baciloscopia direta do escarro, e somente 1 paciente foi excluído por não ter completado a entrevista. Todos os 199 pacientes incluídos procuraram inicialmente $o$ serviço de saúde por iniciativa própria, a partir da percepção pessoal de que estariam ou poderiam estar doentes. As características demográficas da amostra estudada e os intervalos de tempo dos diferentes momentos entre o início dos sintomas - conforme referido pelos pacientes - e o início do tratamento estão apresentados na Tabela 1. A mediana da idade dos pacientes estudados foi de 35 anos (11: 25-48 anos). Nesta amostra, 87 pacientes (44\%) receberam prescrição de antibióticos inespecíficos antes do diagnóstico de tuberculose, sendo que 42 (48\%), 17 (20\%) e $10(11 \%)$, respectivamente, relataram haver utilizado amoxicilina, quinolona respiratória e sulfa, enquanto 18 (21\%) não souberam informar o nome do antibiótico. Na análise por regressão linear, as variáveis gênero, idade, escolaridade, uso prévio de antibióticos, status HIV, local da primeira consulta médica e extensão radiológica da doença não foram associadas a maior tempo para o diagnóstico ou para o início do tratamento.

Os principais motivos descritos pelos pacientes para a demora em procurar atendimento médico foram os seguintes: "achar que os sintomas não indicavam nenhuma doença"; "custo para o deslocamento até uma unidade de saúde"; "dificuldade em conseguir atendimento"; "falta de tempo disponível para procurar uma unidade de saúde"; e "achar que melhorariam espontaneamente”, em 77 (38,7\%), 32 (16,1\%), 29 (14,6\%), $25(12,6 \%)$ e $23(11,6 \%)$, respectivamente, enquanto 13 pacientes $(6,5 \%)$ não apresentaram nenhum motivo.

Em relação ao número de consultas médicas antes do diagnóstico de tuberculose, foram relatadas uma, duas e três consultas, respectivamente, por 61 (31\%), 59 (30\%) e 79 (39\%) pacientes, com mediana de duas consultas (11: 1-3), e 137 pacientes (69\%) procuraram um serviço de emergência para o primeiro atendimento médico. Desses 137 pacientes, os sintomas referidos foram tosse, presença de sangue no escarro, febre, sudorese noturna e perda de peso em 135 (98,5\%), 52 (38,5\%), $112(82,0 \%), 114(83,0 \%)$ e $130(95,0 \%)$, respectivamente.

\section{Discussão}

$\mathrm{Na}$ amostra avaliada, o tempo entre o início dos sintomas, referido pelos pacientes, e o início do tratamento da tuberculose com resultado positivo na baciloscopia do escarro foi longo (11 semanas), sendo a maior parte do período (8 semanas) consequência da demora do paciente em procurar atendimento médico, embora o tempo entre a entrada do paciente no sistema de saúde (primeiro atendimento em uma unidade de 
Tabela 1 - Características dos pacientes, tempo entre o surgimento de sintomas e a procura por atendimento e tempo no sistema de saúde (tempo até o diagnóstico de tuberculose e tempo até o início do tratamento) na amostra estudada. ${ }^{2}$

\begin{tabular}{|c|c|c|c|c|c|c|c|}
\hline \multirow[t]{2}{*}{ Características } & \multirow{2}{*}{$\begin{array}{c}\text { Pacientes, } \\
n\end{array}$} & \multirow{2}{*}{$\begin{array}{c}\text { Tempo anterior à } \\
\text { entrada no sistema } \\
\text { de saúde } \\
\text { Tempo entre } \\
\text { sintomas e procura } \\
\text { por atendimento, } \\
\text { semanas }\end{array}$} & \multirow[t]{2}{*}{$\mathrm{p}$} & \multicolumn{3}{|c|}{ Tempo no sistema de saúde } & \multirow[t]{2}{*}{$\mathrm{p}$} \\
\hline & & & & $\begin{array}{l}\text { Tempo até o } \\
\text { diagnóstico, } \\
\text { semanas }\end{array}$ & $p$ & $\begin{array}{c}\text { Tempo até } \\
\text { o início do } \\
\text { tratamento, } \\
\text { semanas }\end{array}$ & \\
\hline Amostra total & 199 & $8(4-20)$ & & $2(1-8)$ & & $1(1-1)$ & \\
\hline Gênero feminino & 64 & $12(5,5-21)$ & 0,06 & $3,0(1,0-8,0)$ & 0,44 & $1(1-1)$ & 0,41 \\
\hline Gênero masculino & 135 & $8(4-16)$ & & $2,0(1,0-7,5)$ & & $1(1-1)$ & \\
\hline Grau de instrução, anos & 194 & & 0,34 & & 0,16 & & 0,20 \\
\hline$<5$ & 90 & $12(6-16)$ & & $3,5(1-8)$ & & $1(1-1)$ & \\
\hline$\geq 5$ & 104 & $8(4-20)$ & & $2,0(1-7)$ & & $1(1-1)$ & \\
\hline Status HIV & 194 & & 0,08 & & 0,21 & & 0,18 \\
\hline Positivo & 12 & $14(9-30)$ & & $4,5(2,0-27,0)$ & & $1(1-1)$ & \\
\hline Negativo & 182 & $8(4-16)$ & & $2,0(1,0-7,5)$ & & $1(1-1)$ & \\
\hline Uso prévio de antibiótico & 199 & & 0,52 & & 0,35 & & 0,71 \\
\hline Não & 112 & $8(4-20)$ & & $2,0(1,0-8,0)$ & & $1(1-1)$ & \\
\hline Sim & 87 & $8(4-16)$ & & $3,0(1,5-7,0)$ & & $1(1-1)$ & \\
\hline Apresentação radiológica & 182 & & 0,38 & & 0,58 & & 0,67 \\
\hline Doença limitada & 54 & $8(4-17)$ & & $2(1-8)$ & & $1(1-1)$ & \\
\hline Doença extensa & 128 & $8(4-16)$ & & $3(1-8)$ & & $1(1-1)$ & \\
\hline
\end{tabular}

${ }^{a}$ Valores expressos em mediana (intervalo interquartílico).

saúde) e o início do tratamento para tuberculose (3 semanas) também tenha sido excessivo.

Em uma revisão sistemática, mostrou-se que o tempo total até o diagnóstico de tuberculose pulmonar com baciloscopia ou cultura de escarro positiva foi semelhante ao encontrado em nossa amostra. ${ }^{(8)}$ Embora já tenha sido demonstrado em alguns estudos que a duplicação do produto interno bruto per capita está associada à redução significativa na incidência de tuberculose, não houve uma associação entre o tempo até o diagnóstico e o nível socioeconômico nos países nos quais esses estudos foram realizados. ${ }^{(8,9)}$ Uma possível explicação seria o fato de que a tuberculose tende a ser mais prevalente nos segmentos da sociedade com piores marcadores sociais, independentemente da situação econômica média do país. 0 Brasil, por exemplo, tem uma renda per capita de mais de 8.000 dólares/ano e uma taxa média de incidência de tuberculose de 45/100.000 habitantes. No entanto, algumas cidades, como Rio de Janeiro e Nova lguaçu, têm taxas de incidência bem mais elevadas (73/100.000 e 112/100.000 habitantes, respectivamente) e, a despeito da elevada renda média no Brasil, aproximadamente 49 milhões de brasileiros têm renda per capita de até meio salário mínimo por mês, e cerca de 54 milhões não possuem rendimentos. Uma vez que as disparidades socioeconômicas são explícitas entre regiões, estados, cidades e até mesmo entre bairros da mesma cidade, a avaliação das taxas de incidência de tuberculose em paralelo com os índices de desenvolvimento das respectivas áreas poderia fornecer informações relevantes e úteis para o controle da tuberculose. ${ }^{(10-12)}$

$\mathrm{Na}$ amostra estudada, a demora pela procura de uma unidade de saúde foi o principal fator associado ao retardo no diagnóstico e, consequentemente, ao retardo no início do tratamento da tuberculose pulmonar. Esse dado é semelhante aos encontrados em estudos realizados na Etiópia, na Nigéria e em Bangladesh, nos quais o tempo entre o início dos sintomas e o diagnóstico da doença variou entre 7,1 e 11 semanas. ${ }^{(13-15)}$ Os fatores referidos pelos pacientes como justificativa para a demora em procurar o serviço de saúde sugerem que a dificuldade no reconhecimento de sintomas que sugerissem o "adoecimento" (e não especificamente a tuberculose) foi o principal 
fator. Esses achados diferem daqueles de estudos semelhantes realizados em diferentes regiões do mundo, nos quais a falta de conhecimento específico sobre a tuberculose foi a principal justificativa. ${ }^{(9,13,14,16)}$ De fato, na amostra estudada, $39 \%$ dos pacientes relataram não saber que os seus sintomas representavam qualquer doença, e $11,5 \%$ relataram não se sentirem doentes o suficiente para procurar uma unidade de saúde.

As estratégias de busca de casos de tuberculose utilizadas são a busca ativa de casos (BAC), definida como aquela em que o sistema de saúde ativamente procura e identifica o caso de tuberculose antes que esse procure a unidade de saúde por conta própria, e a busca passiva de casos (BPC), que corresponde àquela em que os pacientes procuram unidades de saúde (de nível primário, secundário ou terciário) espontaneamente por se sentirem doentes. $^{(2,3,17)} 0$ presente estudo corresponde a uma amostra de BPC, já que todos os pacientes se dirigiram espontaneamente às unidades de saúde em função de se sentirem doentes. A BPC foi preconizada durante anos pela OMS como parte das recomendações da estratégia de tratamento diretamente observado. ${ }^{(18)}$ No entanto, por ser dependente da capacidade do indivíduo de avaliar seu próprio bem-estar e saúde, assim como do reconhecimento precoce de sinais e sintomas que indiquem uma doença, a BPC pode ter resultados limitados. Os resultados do presente estudo parecem corroborar essa limitação. 0 fato de que os 199 pacientes incluídos no estudo tivessem doença avançada, conforme demonstrado pelo resultado positivo para BAAR no escarro em 100\% dos pacientes e pela presença de doença radiologicamente extensa em 70\% desses, sugere fortemente que esses pacientes estivessem doentes e sintomáticos por um período de tempo significativamente superior às 8 semanas por eles referidas. Em um estudo realizado em uma unidade de saúde no Rio de Janeiro, mostrou-se que 50\% dos pacientes com diagnóstico de tuberculose pulmonar referiam tosse há menos de 2 semanas, embora $80 \%$ deles apresentassem baciloscopia direta do escarro positiva, ou seja, com doença avançada e transmissível. ${ }^{(19)}$ Assim, esse achado já sugeria que o tempo de tosse relatado pelo paciente não seria um indicador apropriado para definir o caso de um paciente que procura uma unidade de saúde como suspeito de ter tuberculose, a despeito das recomendações da OMS e do Programa Nacional de Controle da Tuberculose. ${ }^{(19)}$

Outra causa de demora na procura pelo serviço de saúde, identificada por 12,6\% dos pacientes estudados, foi a dificuldade de acesso à unidade de saúde, dado semelhante ao de estudos realizados em outros países. ${ }^{(13,14,18)} 0$ fato de que $70 \%$ dos pacientes do presente estudo tenham procurado serviços de emergência inicialmente ao invés de uma UPS parece corroborar a ideia de que fatores associados ao acesso e à receptividade nas UPS possam ter uma influência no tempo prolongado até o diagnóstico da tuberculose. Embora as razões da escolha do paciente não tenham sido estudadas na presente amostra, podemos inferir que tal preferência esteja associada à rapidez no atendimento e à possibilidade de realização de exames complementares, como exames radiológicos, nos serviços de emergência. Um problema adicional na procura do paciente com suspeita de tuberculose pelo serviço de emergência é a ausência de medidas de biossegurança para a prevenção da tuberculose, assim como a impossibilidade de realização da pesquisa direta para BAAR nessas unidades. Já foi descrito que os serviços públicos de saúde raramente são considerados apropriados nas áreas e regiões nas quais a tuberculose é mais prevalente e incidente, prejudicando ainda mais a efetividade da estratégia de BPC. ${ }^{(15,20)}$

Devido a vários fatores, entre os quais os decepcionantes resultados da estratégia BPC, em 2006, o Segundo Plano Global para Eliminar a Tuberculose do Mundo da OMS incorporou as estratégias BAC e de busca intensificada de casos de tuberculose, que demandam um esforço especial do sistema de saúde, com o objetivo de aumentar a detecção de casos de tuberculose em grupos da população considerados com maior risco de tuberculose (contatos de tuberculose, indivíduos que vivem em áreas com taxa de incidência muito elevada, entre outros). ${ }^{(21)}$ Nesse contexto, o objetivo da BAC e da busca intensificada de casos é diagnosticar e tratar a tuberculose pulmonar antes que o paciente procure o serviço de saúde por iniciativa própria, quebrando, assim, a cadeia de transmissão, reduzindo o número de casos de contatos infectados e, consequentemente, reduzindo o número de casos de tuberculose ativa no futuro.

A média do tempo referido pelos pacientes entre a primeira consulta em uma unidade de saúde e o diagnóstico de tuberculose foi 
de 2 semanas, com até $25 \%$ dos casos sendo diagnosticados e tendo o tratamento iniciado em um intervalo de tempo total de 8 semanas (11: 1-8 semanas). Certos fatores, como a sobrecarga do sistema de saúde e a falta de conhecimentos sobre tuberculose por parte dos profissionais de saúde, têm sido apontados como associados ao atraso no diagnóstico da tuberculose pulmonar, inclusive por parte dos gestores do sistema de saúde. ${ }^{(15,22,23)} \mathrm{Na}$ amostra estudada, os pacientes tiveram, em média, duas consultas médicas até o diagnóstico, e 58 pacientes (29\%) tiveram o diagnóstico realizado com apenas uma consulta médica, demonstrando o elevado grau de suspeição de tuberculose por parte dos médicos. No entanto, somente pacientes com resultados positivos para BAAR na pesquisa direta do escarro foram incluídos no estudo, e esse é um procedimento que leva apenas algumas horas para ser realizado. Assim, podemos considerar o tempo para o diagnóstico ( 2 semanas) como inaceitavelmente longo. 0 tempo entre a solicitação de uma baciloscopia direta do escarro em um paciente com suspeita de tuberculose e capaz de expectorar espontaneamente (como foi o caso na amostra estudada) e a liberação do resultado (com consequente conhecimento do mesmo por parte da equipe de saúde) é de, aproximadamente, $24 \mathrm{~h}$. Além disso, em função da ausência de um local apropriado para a coleta de escarro na maioria das UPS, muitas vezes o paciente é orientado a coletar o escarro em casa e retornar no dia seguinte, aumentando ainda mais o tempo necessário até o diagnóstico e o custo do procedimento para o paciente. Esses achados sugerem que o retardo no diagnóstico de tuberculose após a entrada do paciente no sistema de saúde esteja mais associado a problemas de infraestrutura do sistema de saúde do que ao tempo de realização do teste diagnóstico ou à falta de conhecimentos dos profissionais da área de saúde.

Da mesma forma, o tempo entre o resultado da baciloscopia e o inicio do tratamento para a tuberculose ( 1 semana) não é adequado. 0 tempo entre o resultado positivo de uma baciloscopia e o início do tratamento não deve ultrapassar 24-48 h.

0 presente estudo tem várias e importantes limitações. Por ter sido realizado em apenas uma UPS, seus achados e conclusões podem não ser aplicáveis a outras UPS ou regiões. No entanto, em um estudo recente, no qual se avaliou o tempo entre o início dos sintomas e o diagnóstico de tuberculose em 20 unidades básicas de saúde e 3 unidades hospitalares do estado do Rio de Janeiro, os dados foram semelhantes aos nossos, a despeito de a amostra do referido estudo ter sido mais heterogênea. ${ }^{(24)}$ Outra limitação é o critério diagnóstico de tuberculose utilizado no presente estudo (presença de BAAR no escarro). Embora a baciloscopia direta tenha elevado valor preditivo positivo em nosso meio, o diagnóstico de certeza de tuberculose somente é feito com a cultura positiva para $M$. tuberculosis. Além disso, a inclusão somente de pacientes com BAAR positivo no escarro pode se constituir em um viés de seleção para pacientes HIV positivos, não permitindo uma análise adequada do impacto da infecção por HIV nos tempos de diagnóstico e de tratamento. Também, o fato de a amostra ter sido constituída, em sua maioria, por pacientes do gênero masculino (135/199) pode não ter permitido a avaliação apropriada do impacto do gênero nos tempos até o diagnóstico e até o tratamento da tuberculose. Deve também ser considerado que a extensão de doença, avaliada como uma variável preditora, pode, na verdade, ter sido uma consequência do tempo até o diagnóstico. Alguns dados do presente estudo (tempo de sintomas, primeira consulta médica, etc.) foram obtidos através de entrevista, o que pode introduzir um viés de informação no estudo.

Em conclusão, os tempos até o diagnóstico e até o início do tratamento para tuberculose foram longos na amostra estudada. A principal causa para o retardo no diagnóstico da tuberculose foi a apresentação tardia do paciente ao serviço de saúde, tanto pela falta de percepção de estar doente quanto pela dificuldade de acesso às unidades de saúde. Essa demora sugere a necessidade da implementação de estratégias que visem o diagnóstico precoce da tuberculose, sobretudo em áreas e em grupos de elevado risco da doença. Os longos tempos, tanto entre a entrada do paciente no sistema de saúde e o diagnóstico de tuberculose quanto entre a baciloscopia de escarro positiva e o início do tratamento, sugerem a necessidade de estratégias que reduzam esses intervalos de tempo, como, por exemplo, o investimento em estrutura e em informatização do sistema de saúde. 


\section{Referências}

1. World Health Organization [homepage on the Internet]. Geneva: World Health Organization [cited 2010 Mar 1]. Global Tuberculosis Control 2010. [Adobe Acrobat document, 218p.]. Available from: http://whqlibdoc. who.int/publications/2010/9789241564069_eng.pdf

2. Portal da Saúde [homepage on the Internet]. Brasília: Ministério da Saúde. [cited 2010 Mar 1]. Manual de Recomendações para o Controle da Tuberculose no Brasil. [Adobe Acrobat document, 186p.]. Available from: http://portal.saude.gov.br/portal/arquivos/pdf/ manual_de_recomendacoes_controle_tb_novo.pdf

3. Conde MB, Melo FA, Marques AM, Cardoso NC, Pinheiro VG, Dalcin Pde T, et al. 111 Brazilian Thoracic Association Guidelines on tuberculosis. J Bras Pneumol. 2009;35(10):1018-48. PMid:19918635.

4. Golub JE, Bur S, Cronin WA, Gange S, Baruch N, Comstock GW, et al. Delayed tuberculosis diagnosis and tuberculosis transmission. Int J Tuberc Lung Dis. 2006;10(1):24-30. PMid:16466033.

5. Job JR, Gozzano JO, Bernardes Júnior OR, Garcia RH, Miralhes 0J, de Miranda MA. Data preceding the diagnosis of pulmonary tuberculosis and time elapsed till the beginning of treatment in patients enrolled at a health center, São Paulo (Brazil) [Article in Portuguese]. Rev Saude Publica. 1986;20(1):21-5. http://dx.doi.org/10.1590/ S0034-89101986000100002

6. Ruffino-Netto A. Controle da tuberculose no Brasil: dificuldades na implantação do programa. J Pneumol. 2000;26(4):159-62. http://dx.doi.org/10.1590/ S0102-35862000000400001

7. Ruffino-Netto A. Tuberculosis: the neglected calamity [Article in Portuguese]. Rev Soc Bras Med Trop. 2002;35(1):51-8. PMid:11873262. http://dx.doi. org/10.1590/S0037-86822002000100010

8. Sreeramareddy CT, Panduru KV, Menten J, Van den Ende J. Time delays in diagnosis of pulmonary tuberculosis: a systematic review of literature. BMC Infect Dis. 2009;9:91. PMid:19519917. PMCid:2702369. http:// dx.doi.org/10.1186/1471-2334-9-91

9. Janssens JP, Rieder HL. An ecological analysis of incidence of tuberculosis and per capita gross domestic product. Eur Respir J. 2008;32(5):1415-6. PMid:18978146. http:// dx.doi.org/10.1183/09031936.00078708

10. Instituto Brasileiro de Geografia e Estatística [homepage on the Internet]. Brasília: Governo do Brasil. [cited 2011 Jun 1] Available from: www.ibge.com.br

11. Secretaria de Estado de Saúde [homepage on the Internet]. Rio de Janeiro: Secretaria de Estado de Saúde. [cited 2011 Jul 1]. Vigilância em Saúde - Vigilância Epidemiológica e Ambiental - Boletim Epidemiológico e Ambiental 2009 / 2010 Available from: http://www.saude.rj.gov. $\mathrm{br} /$ servicos-vigiliancia-em-saude/vigilancia-em-saudevigilancia-epidemiologica-e-ambiental/6482-boletimepidemiologico-e-ambiental-2009-2010

12. Prefeitura Municipal de Nova lguaçu [homepage on the Internet]. Nova Iguaçu: Prefeitura Municipal de Nova lguaçu. [cited 2011 Mar 1]. Apresentação. Available from: http://www.novaiguacu.rj.gov.br/apresentacao.php
13. Demissie M, Lindtjorn B, Berhane Y. Patient and health service delay in the diagnosis of pulmonary tuberculosis in Ethiopia. BMC Public Health. 2002;2:23. PMid:12296975. PMCid:130033. http://dx.doi.org/10.1186/1471-2458-2-23

14. Odusanya 00, Babafemi J0. Patterns of delays amongst pulmonary tuberculosis patients in Lagos, Nigeria. BMC Public Health. 2004;4:18. PMid:15169548. PMCid:434509. http://dx.doi.org/10.1186/1471-2458-4-18

15. Karim F, Islam MA, Chowdhury AM, Johansson E, Diwan VK. Gender differences in delays in diagnosis and treatment of tuberculosis. Health Policy Plan. 2007;22(5):32934. Erratum in: Health Policy Plan. 2008;23(6):476. PMid:17698889. http://dx.doi.org/10.1093/heapol/czm026

16. World Health Organization - Regional Office for the Eastern Mediterranean [homepage on the Internet]. Cairo: World Health Organization. [cited 2011 Jul 1]. Diagnostic and treatment delay in tuberculosis. [Adobe Acrobat document, 48p.]. Available from: http://www. emro.who.int/dsaf/dsa710.pdf

17. Golub JE, Mohan $\mathrm{Cl}$, Comstock GW, Chaisson RE. Active case finding of tuberculosis: historical perspective and future prospects. Int J Tuberc Lung Dis. 2005;9(11):1183203. PMid:16333924.

18. World Health Organization [homepage on the Internet]. Geneva: World Health Organization. [cited 2011 Jul 1]. Global tuberculosis control: Surveillance, planning, financing. WHO Report 2002. Available from: http:// www.who.int/tb/publications/global_report/2002/en/ index.html

19. Bastos LG, Fonseca LS, Mello FC, Ruffino-Netto A, Golub JE, Conde MB. Prevalence of pulmonary tuberculosis among respiratory symptomatic subjects in an out-patient primary health unit. Int J Tuberc Lung Dis. 2007;11(2):15660. PMid:17263285.

20. Jaramillo E. Pulmonary tuberculosis and health-seeking behaviour: how to get a delayed diagnosis in Cali, Colombia. Trop Med Int Health. 1998;3(2):138-44. PMid:9537276. http://dx.doi.org/10.1046/j.1365-3156.1998.00202.x

21. World Health Organization [homepage on the Internet]. Geneva: World Health Organization. [cited 2011 Jul 1]. The Stop TB Strategy - Building on and enhancing DOTS to meet the TB-related Millennium Development Goals. [Adobe Acrobat document, 24p.]. Available from: http://www.who.int/tb/publications/2006/who_htm_ tb_2006_368.pdf

22. Kiwuwa MS, Charles K, Harriet MK. Patient and health service delay in pulmonary tuberculosis patients attending a referral hospital: a cross-sectional study. BMC Public Health. 2005;5:122. PMid:16307685. PMCid:1310609. http://dx.doi.org/10.1186/1471-2458-5-122

23. Xu B, Jiang QW, Xiu Y, Diwan VK. Diagnostic delays in access to tuberculosis care in counties with or without the National Tuberculosis Control Programme in rural China. Int J Tuberc Lung Dis. 2005;9(7):784-90. PMid:16013775.

24. Machado AC, Steffen RE, Oxlade O, Menzies D, Kritski A, Trajman A. Factors associated with delayed diagnosis of pulmonary tuberculosis in the state of Rio de Janeiro, Brazil. J Bras Pneumol. 2011;37(4):512-20. PMid:21881742. http://dx.doi.org/10.1590/S1806-37132011000400014 


\section{Sobre os autores}

\section{Marina de Loureiro Maior}

Mestranda em Pneumologia. Universidade Federal do Rio de Janeiro, Rio de Janeiro (RJ) Brasil.

\section{Renata Leborato Guerra}

Doutoranda em Pneumologia. Universidade Federal do Rio de Janeiro, Rio de Janeiro (RJ) Brasil.

\section{Michelle Cailleaux-Cezar}

Doutoranda em Pneumologia. Universidade Federal do Rio de Janeiro, Rio de Janeiro (RJ) Brasil.

\section{Jonathan Eric Golub}

Epidemiologista. Johns Hopkins University, Baltimore (MD) EUA.

\section{Marcus Barreto Conde}

Professor Associado. Universidade Federal do Rio de Janeiro, Rio de Janeiro (RJ) Brasil. 